\title{
How I do it - CT-Protokolle beim akuten Abdomen
}

Kristina I. Ringe, Andreas G. Schreyer

\section{HINWEIS}

Die Beiträge in der Rubrik „How I do it“ sind als Anregung und Orientierungshilfe zu verstehen. Es bleibt jeder/jedem Kollegin/Kollegen überlassen, von diesem Schema abzuweichen, sofern man sich mit der Indikationsfrage befasst hat und sachliche Gründe für ein alternatives Vorgehen sprechen.

\section{Einleitung}

Beim akuten Abdomen handelt es sich um die Manifestation von Erkrankungen im Bauchraum, die einer sofortigen Diagnostik und Therapie bedürfen. Leitsymptome sind der (akut auftretende) abdominelle Schmerz, eine Störung der Darmperistaltik mit Übelkeit und Erbrechen sowie Kreislaufstörungen bis zum Schock [1]. In mehr als 90\% der Fälle ist die Ursache auf 8 Krankheitsbilder zurückzuführen: Appendizitis, Cholezystitis, Dünndarmileus, gynäkologische Erkrankungen, akute Pankreatitis, Nierenkolik, perforiertes Ulkus, Sigmadivertikulitis [2]. Die genaue Verdachtsdiagnose basiert dabei im Wesentlichen auf der individuellen Patientenanamnese, der klinischen Untersuchung und den Ergebnissen von Labortests.

Zur Bestätigung bzw. zum Ausschluss der entsprechenden Arbeitsdiagnose spielt die weitere Bildgebung eine entscheidende Rolle. Die CT ist in der Regel die bildgebende Methode der Wahl zur Abklärung von Patienten mit einem akuten Abdomen. Dies liegt vor allem an der mittlerweile sehr guten Verfügbarkeit, schnellen Bildakquisition und hohen diagnostischen Genauigkeit mit signifikantem Einfluss auf die weitere Behandlung [3].

\section{Aktueller Wissensstand}

Derzeit gibt es in Deutschland keine einheitlichen Empfehlungen zur CT-diagnostischen Abklärung des akuten Abdomens. Der Stellenwert der CT in diesem Szenario wird dennoch zweifellos durch eine ganze Reihe an Studien untermauert [4, 5]. Insbesondere mit Blick auf die internationale Literatur wird die höchste Sensitivität erzielt, wenn Patienten mit akuten abdominellen Schmerzen zunächst sonografisch abgeklärt werden und die CT nur bei negativen bzw. bei unklaren Befunden ergänzt wird (nur
6\% falsch negative Ergebnisse) [3]. Die Heterogenität der dabei im klinischen Alltag verwendeten Untersuchungsprotokolle (Art der Kontrastierung, Kontrastmittelphasen usw.) ist in einer kürzlich durchgeführten Umfrage der Arbeitsgemeinschaft Gastrointestinal- und Abdominaldiagnostik der Deutschen Röntgengesellschaft gezeigt worden [6]. Der potenzielle Zugewinn der neusten CT-Weiterentwicklung in Form der Dual-Energy-CT (DECT) ist vielversprechend und Gegenstand aktueller Untersuchungen [7].

\section{Vorgehensweise}

\section{Diagnostischer Algorithmus}

Ein differenzierter diagnostischer Algorithmus bei Patienten mit einem akuten Abdomen ist in $\mathbf{A} \mathbf{A b} \mathbf{b} \mathbf{1}$ dargestellt. Die Sonografie kann bereits ein breites Spektrum an Differenzialdiagnosen abdecken, insbesondere, wenn es um Veränderungen an der Gallenblase geht. Die Abdomenübersichtsaufnahme ist zwar in vielen Bereichen durch die CT weitestgehend abgelöst, kann aber in der Akutphase - insbesondere in der Notaufnahme - nicht selten als erste Orientierung, beispielsweise zum Ausschluss freier Luft, eines lleus oder von Fremdkörpern hilfreich sein. Die Datenlage zum Einsatz der MRT beim akuten Bauchschmerz ist im Vergleich zu den anderen Modalitäten noch relativ spärlich. Aufgrund der allgemein etwas schlechteren Verfügbarkeit und längeren Untersuchungsdauer ist diese bei den zum Teil klinisch stark beeinträchtigten Patienten in der Regel speziellen Situation vorbehalten (z. B. der Untersuchung von Schwangeren oder Kindern) [8]. Die CT ist die Bildgebung der Wahl und das Arbeitspferd bei der weiteren Abklärung von Patienten mit einem akuten Abdomen.

\section{Untersuchungsprotokolle}

Bei der CT von Patienten mit einem akuten Abdomen ist ein an die klinische Verdachtsdiagnose angepasstes Untersuchungsprotokoll zu empfehlen ( $\triangleright$ Abb. 1, Tab.1). Sofern keine Kontraindikationen bestehen, sollte die Untersuchung eine i.v. Kontrastmittelgabe einschließen. Insbesondere in der Notfallsituation ist dabei eine entsprechende Nutzen-Risiko-Abwägung erforderlich. Eine Ausnahme ist die Bildgebung bei Verdacht auf Urolithiasis, hier ist ein natives Low-Dose-Protokoll ausreichend ( Tab. 1, Tab. 2). 


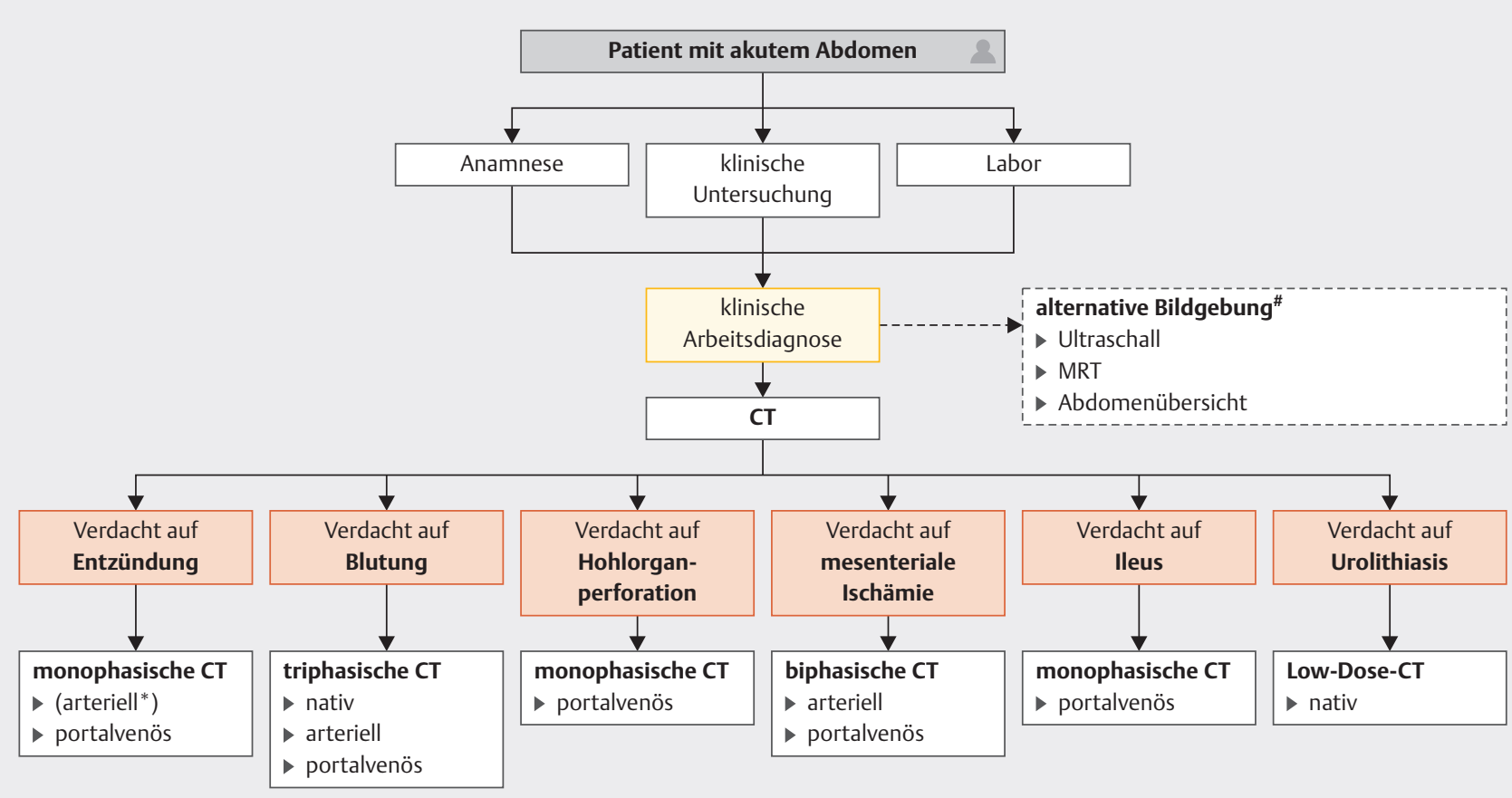

- Abb. 1 Diagnostischer Algorithmus bei Patienten mit einem akuten Abdomen; \# MRT oder Ultraschall ggf. primär bei Kindern und Schwangeren erwägen bzw. in Abhängigkeit der genauen Fragestellung (z. B. Ultraschall bei Verdacht auf Cholezystitis); ${ }^{*}$ zusätzlich arterielle Phase über den Oberbauch bei Verdacht auf Pankreatitis.

- Tab. 1 Untersuchungsprotokolle für die CT beim akuten Abdomen (ab 64-Zeiler aufwärts).

\begin{tabular}{|l|l|l|l|l|l|}
\hline Phase & Kontrastmittelmenge & Injektionsrate & Triggerort & Triggerschwelle & Delay (s) \\
\hline nativ & - & - & - & - & - \\
\hline arteriell & $88 \mathrm{ml}$ & $3,5 \mathrm{ml} / \mathrm{s}$ & Aorta descendens & $150 \mathrm{HU}$ & 10 \\
\hline portalvenös & $88 \mathrm{ml}$ & $3,5 \mathrm{ml} / \mathrm{sec}$ & Milz & $150 \mathrm{HU}$ & 20 \\
\hline \begin{tabular}{l} 
biphasisch (arteriell plus portalvenös): Interscan Delay $30 \mathrm{~s} ; \mathrm{HU}=$ Hounsfield-Einheiten \\
\hline
\end{tabular}
\end{tabular}

- Tab. 2 Scanparameter für die CT beim akuten Abdomen (ab 64-Zeiler aufwärts).

\begin{tabular}{|l|l|l|l|l|}
\hline Phase & Röhrenspannung $\mathbf{( k V )}$ & Röhrenstrom (mAs) & Pitch & Schichtdicke (mm) \\
\hline nativ Low-dose & 120 & bis 300 & $0,6-1$ & $1-1,25$ \\
\hline nativ & 120 & ATM & $0,6-1$ & $1-1,25$ \\
\hline arteriell & 120 & ATM & $0,6-1$ & $1-1,25$ \\
\hline portalvenös & 120 & ATM & $0,6-1$ & $1-1,25$ \\
\hline
\end{tabular}

sofern möglich, sollten zusätzlich iterative Rekonstruktionsalgorithmen zwecks Dosisreduktion angewendet werden; ATM = automatische Röhrenstrommodulation 
Eine ergänzende enterale Kontrastmittelgabe (oral/rektal) ist im Allgemeinen nicht zu empfehlen. In der Regel verzögert sie die Diagnostik unnötig und führt u.U. zu einer Verschlimmerung der klinischen Symptomatik (z. B. bei Patienten mit lleus). Ein diagnostischer Zugewinn ist meist nicht zu erwarten [9]. Darüber hinaus können insbesondere bei Verwendung positiver Kontrastmittel entscheidende Befunde maskiert werden (z.B. Darmschleimhaut).

\section{Fazit}

Die radiologische Bildgebung in Form der CT spielt eine zentrale Rolle bei der Abklärung des akuten Abdomens, insbesondere in Ergänzung zu einer eventuell vorausgegangenen Sonografie. Das Untersuchungsprotokoll sollte, wenn möglich, an die klinische Verdachtsdiagnose angepasst sein. Bis auf die Abklärung der Urolithiasis ist eine i.v. Kontrastmittelgabe indiziert, von einer ergänzenden enteralen Kontrastierung ist abzusehen.

\section{Interessenkonflikt}

Die Autorinnen/Autoren geben an, dass kein Interessenkonflikt besteht.

\section{Autorinnen/Autoren}

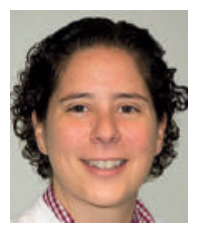

\section{Kristina I. Ringe}

Prof. Dr. med. 1997-2004 Studium der Humanmedizin an der Georg-August Universität Göttingen. 2006 Promotion. 2004-2010 Weiterbildung zur Fachärztin für Radiologie, Medizinische Hochschule Hannover. 20092010 Research Fellowship, Department of Radiology, Duke University Medical Center, Durham, USA. 2014 Habilitation. Seit 2012 Oberärztin Institut für Diagnostische und Interventionelle Radiologie, Medizinische Hochschule Hannover, Bereichsleitung Abdominelle Radiologie.

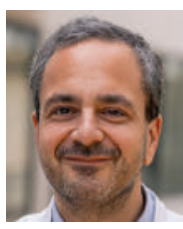

\section{Andreas G. Schreyer}

Univ.-Prof. Dr. med. 1990-1996 Studium der Humanmedizin an der Friedrich-AlexanderUniversität Erlangen/Nürnberg und Universität Regensburg. 1996-1997 AiP Klinikum Neuperlach/München, Chirurgische Abteilung. 1998 Promotion. 1997-1999 MRI Research Fellowship am Brigham \& Women's Hospital der Harvard Medical School Boston. 1999-2006 Weiterbildung zum Facharzt Radiologie Universitätsklinikum Regensburg. 2006-2010 Oberarzt und geschäftsführender Oberarzt Universitätsklinikum Regensburg. 2010-2019 Stellv. Institutsdirektor und leitender Oberarzt am Institut für Röntgendiagnostik Universitätsklinikum Regensburg und gleichzeitig 2018 kommissarischer Chefarzt Donau-Isar-Klinikum Deggendorf. Seit 2019 Direktor und Chefarzt des Instituts für Diagnostische und Interventionelle Radiologie an der Medizinischen Hochschule Brandenburg (MHB) am Klinikum Brandenburg.

\section{Prof. Dr. med. Kristina I. Ringe}

Medizinische Hochschule Hannover

Institut für Diagnostische und Interventionelle Radiologie

Carl-Neuberg-Straße 1

30625 Hannover

ringe.kristina@mh-hannover.de

\section{Literatur}

[1] Reng, CM, Langgartner J. Akutes Abdomen - Strategien zur präklinischen und primären klinischen Versorgung einer „interdisziplinären Erkrankung“. J Intensivmed 2003; 49: 599617

[2] De Dombal FT. The diagnosis of acute abdominal pain with computer assistance: worldwide perspective. Ann Chir 1991; 45: $273-277$

[3] Lameris W, van Randen A, van Es HW et al. Imaging strategies for detection of urgent conditions in patients with acute abdominal pain: diagnostic accuracy study. BMJ 2009; 338: b2431

[4] van Randen A, Lameris W, van Es HW et al. A comparison of the accuracy of ultrasound and computed tomography in common diagnoses causing acute abdominal pain. Eur Rad 2011; 21: 1535-1545

[5] Sala E, Watson C], Beadsmoore C et al. A randomized, controlled trial of routine early abdominal computed tomography in patients presenting with non-specific acute abdominal pain. Clin Rad 2007; 62: 961-969

[6] Schreyer AG, Wessling J, Grenacher L. Versorgungsrealität vs leitliniengerechte Bildgebung in der Abdominalradiologie im deutschsprachigen Raum: Ergebnisse einer Online-Umfrage. Fortschr Röntgenstr 2016; 188: 268-279

[7] Murray N, Darras KE, Walstra FE et al. Dual-Energy CT in Evaluation of the Acute Abdomen. Radiographics 2019; 39: 264286

[8] Mushtaq R, Desoky SM, Morello F et al. First-Line Diagnostic Evaluation with MRI of Children Suspected of Having Acute Appendicitis. Radiology 2019; 291: 170-177

[9] Kessner R, Barnes S, Halpern P et al. CT for Acute Nontraumatic Abdominal Pain-Is Oral Contrast Really Required? Acad Rad 2017; 24: 840-845

Bibliografie

DOI https://doi.org/10.1055/a-1133-9246

Radiologie up2date 2020; 20: 211-213

(c) Georg Thieme Verlag KG Stuttgart · New York

ISSN 1616-0681 